Portland State University

PDXScholar

Physics Faculty Publications and Presentations

Physics

$1-1-1992$

\title{
Diffraction of light by a focused ultrasonic wave
}

J. Huang

Portland State University

Joel Alan Nissen

Stanford University

Erik Bodegom

Portland State University

Follow this and additional works at: https://pdxscholar.library.pdx.edu/phy_fac

Part of the Physics Commons

Let us know how access to this document benefits you.

\section{Citation Details}

"Diffraction of light by a focused ultrasonic wave," with J. Huang and J. A. Nissen, J. Appl. Phys. 71, 70 (1992).

This Article is brought to you for free and open access. It has been accepted for inclusion in Physics Faculty Publications and Presentations by an authorized administrator of PDXScholar. Please contact us if we can make this document more accessible: pdxscholar@pdx.edu. 


\title{
Diffraction of light by a focused ultrasonic wave
}

\author{
J. Huang \\ Department of Physics and Environmental Sciences and Resources Program, Portland State University, \\ Portland, Oregon 97207 \\ J. A. Nissen \\ Physics Department, Stanford University, Stanford, California 94305 \\ Erik Bodegom \\ Department of Physics and Environmental Sciences and Resources Program, Portland State University. \\ Portland, Oregon 97207
}

(Received 31 May 1991; accepted for publication 26 September 1991)

\begin{abstract}
The measurement of the acoustic pressure of a planar ultrasonic wave by light diffraction is well established. The ability to do similar measurements in the case of spherical waves has been doubted. However, we show that the range of validity can be extended to the focal region of a spherical concave piezoelectric transducer. Light is passed through the focal plane of a spherical concave transducer and is diffracted as a result of the variation in the index of refraction. The peak pressure can be calculated from the diffraction intensity by making the following simplification. We assume that in the focal plane the ultrasound can be approximated by a profiled planar wave, which in turn can be modeled by a wave of constant amplitude and effective width. The experimental results for moderatepressure amplitudes in water compare favorably with the calculations using the KhokhlovZabolotskaya-Kuznetsov equation, which incorporates both nonlinearity and diffraction effects of the acoustic field.
\end{abstract}

\section{INTRODUCTION}

The diffraction of light by ultrasound has received a lot of attention in recent years because of many applications such as light-beam defiection, spectrum analysis, acoustooptic signal processing, ${ }^{1-3}$ and for studying the characteristics of cryogenic liquids. ${ }^{4}$ The theory of acousto-optical interaction for plane waves was well established by Raman and Nath in the 1930s. ${ }^{5}$ Raman and Nath showed that under certain conditions the ultrasound field can be considered as a pure optical-phase grating. The diffraction pattern is given by the well-known Bessel function expression for the individual orders. It was Mertens ${ }^{6}$ who succeeded in working out a generalized approach of the differential equations of the Raman-Nath model. The range of validity was, as a result, greatly extended. Considerable effort has been made to apply the theory of Raman and Nath to a bounded sound field which is treated as profiled planar wave. ${ }^{7-9}$ Leroy and Claeys ${ }^{10}$ developed a model based on a generalization of the generating function method to solve the appropriate Raman-Nath system of equations. They derived the analytical expressions for the intensity of light diffracted by a single-frequency ultrasonic field with nonuniform amplitude distribution.

This study of light diffraction by ultrasound was motivated by cavitation studies of cryogenic liquids in which the ultrasound was highly focused in order to obtain the maximum tensile strength of the liquids. ${ }^{4}$ The advantage of an optical method for detecting ultrasound lies in its ability to do so without disturbing the sound field or introducing nucleation sites. Moreover, the Raman-Nath method provides an absolute measurement of the pressure amplitudes. ${ }^{11,12}$ It has been shown that the Raman-Nath theory, even for small curvature of the phase fronts, leads to large errors. ${ }^{13}$ For focused ultrasonic field, this method needs to be modified.

This paper is organized as follows. We first review the Raman-Nath theory and then describe our modified model to deal with the spherical ultrasonic wave in the focal region. Next, Debye's theory and the KhokhlovZabolotskaya-Kuznetsov (KZK) equation are discussed. We will present the theoretical results from the improved Raman-Nath model and from the KZK equations and compare the resulting pressure amplitudes with the experimentally obtained amplitudes.

\section{THEORY}

\section{A. Diffraction from a profiled beam}

Under the assumption that the index of refraction, $\mu$, in the presence of the ultrasonic field of frequency $\omega^{*}$ can be expressed as

$$
\mu=\mu_{0}+\mu_{1} \sin \left(\omega^{*} t\right),
$$

light diffraction by progressive harmonic waves is well described by the system of difference-differential equations of Raman and Nath. The solution represents the normalized amplitude of the electric field $E_{n}$ of the $n$th diffraction order:

$$
\begin{aligned}
& 2 \frac{d E_{n}}{d \xi}-E_{n-1}+E_{n+1}=i^{2} Q E_{n}, \\
& \xi=\frac{2 \pi \mu_{1} x}{\lambda},
\end{aligned}
$$




$$
Q=\frac{\lambda^{2}}{\mu_{0} \mu_{1} \lambda^{2}},
$$

where $x$ is the position along the direction of light propagation, $\mu_{0}$ is the refractive index of the undisturbed liquid, $\mu_{1}$ is the maximum variation of the refractive index due to the ultrasonic wave of wavelength $\lambda^{*}$, and $\lambda$ is wavelength of light. In the Raman-Nath approximation, the intensity of the $n$ th-order diffraction peak can be shown to be proportional to the square of the nth-order Bessel function: ${ }^{14-16}$

$$
I_{n}(\nu) \propto J_{n}^{2}(\nu) .
$$

$v$ is the Raman-Nath parameter, which can be expressed as

$$
\nu=\frac{2 \pi l}{\lambda}\left(\frac{\partial \mu}{\partial P}\right)_{s},
$$

where $P$ is the acoustic pressure amplitude and the derivative is taken at constant entropy. The parameter $l$ is the light-sound interaction length. The solution given by Eq. (3) is subject to the limitation that $v$ and $Q$ are both small: ${ }^{17}$

$$
\begin{aligned}
& \nu<6, \\
& Q=\frac{\lambda^{2}}{\mu_{0} \mu_{1} \lambda^{* 2}}<1 .
\end{aligned}
$$

When, besides the fundamental frequency, higher harmonics are present, one can express the index of refraction in the ultrasonic field as

$$
\mu=\mu_{0}+\sum_{j=1}^{\infty} a_{j} \mu_{1} \sin \left[j\left(\omega^{*} t-k^{*} r\right)+\phi_{j}\right]
$$

where $a_{j}$ is the ratio of the amplitude of the $j$ th harmonic to the fundamental, $k^{*}$ is the acoustic wave number of the $j$ th harmonic, and $\phi_{j}$ is the phase factor of the $j$ th harmonic relative to the fundamental. If one considers only the fundamental and its second harmonic, the corresponding intensity of diffracted light can be written as ${ }^{18}$

$$
I_{n}(v)=\left|\sum_{k=-\infty}^{\infty} J_{n-2 k}(v) J_{k}\left(a_{2} v\right)\right|^{2} .
$$

In the original Raman-Nath formulation, $l$ is simply the depth of the sound beam. The acoustic pressure amplitude along the light path is assumed to be a step function. When the amplitude of the acoustic field varies greatly along the optical path such as in a profiled beam, the above assumption is no longer valid. In this case, one can postulate an "effective interaction length" which describes the average effect of the changing pressure distribution along the optical path. We take the spatial average of the pressure distribution along the optical path as the new effective interaction length $l^{*}$, which can be written as

$$
I^{*}=\frac{1}{P_{m}} \int_{-\infty}^{+\infty} P(x) d x,
$$

where $P_{m}$ is the maximum pressure amplitude. Note that for a material with index of refraction proportional to the acoustic pressure amplitude, $\int P(x) d x$ is proportional to the change in optical path length caused by the pressure of the sound field.

\section{B. Diffraction of light in the focal region}

In the prefocal region of a converging acoustic wave, the initially spherical wave front gradually flattens out, then passes through the focus, and becomes a diverging spherical wave. The curvature of the wave front experiences a change of sign. Based on the assumption that the radius of wave-front curvature changes continuously and the focus has a finite size, one has the argument that, in the focal region, the focusing spherical wave can be treated as a planar wave with nonuniform amplitude. Its radial distribution in the focal plane can be written, from Debye theory, ${ }^{19-21}$

$$
P(r, t) \doteq P_{f} e^{i\left[\omega^{*} t+\delta(r)\right]} \int_{0}^{\alpha_{m}} J_{0}\left(k^{*} r \sin \alpha\right) \sin \alpha d \alpha,
$$

with the condition

$$
F>\lambda^{*},
$$

and with

$$
P_{f}=G P_{0}=F k^{*}\left(1-\cos \alpha_{m}\right) P_{0} .
$$

$P_{f}$ is the peak pressure amplitude in the focal plane, $\alpha_{m}$ is the half aperture angle of the radiator, $F$ is the focal length of the transducer, $J_{0}(x)$ is the zeroth-order Bessel function, and $\delta(r)$ is a phase factor. $P_{0}$ is the pressure on the surface of the transducer, and $G$ is the gain of the transducer. For $\alpha_{m} \leqslant \pi / 6, \sin \alpha \approx \alpha$ and the time-independent pressure distribution can be approximated as

$$
P(r)=P_{f} \frac{2 J_{1}\left(k r \alpha_{m}\right)}{k r \alpha_{m}} .
$$

If $\alpha_{m}>\pi / 6$, then the integral can be solved by series expansion. In this paper we will only be interested in the transducers with small aperture angles. Thus the effective interaction length can be expressed as

$$
l^{*}=\frac{2}{P_{f}} \int_{0}^{\infty} P(r) d r
$$

Upon substituting Eq. (13) into (14), we get

$$
l^{*}=4 \int_{0}^{\infty} \frac{J_{1}\left(k^{*} \alpha_{m} r\right) d r}{k^{*} r \alpha_{m}}
$$

which gives

$$
l^{*}=\frac{4}{k^{*} \alpha_{m}} \text {. }
$$

Here we have ignored the phase factor $\delta(r)$ in Eq. (10), and we will show later that it has negligible influence on the final result.

Substituting (16) into (4) yields

$$
v=\frac{4 \lambda^{*}}{\alpha_{m} \lambda}\left(\frac{\partial \mu}{\partial P}\right)_{s} P_{f}
$$




\section{The pressure distribution calculated from the KZK equation}

So far, we have neglected nonlinearity and dissipation of the liquids. Directive acoustic beams in which the effects of nonlinearity, diffraction, and dissipation are important may be modeled using the KZK equation which, in dimensionless form, is given by ${ }^{22}$

$$
\begin{aligned}
& \frac{\partial^{2} P^{\prime}}{\partial \tau \partial \sigma}-\frac{1}{4} \nabla_{\perp}^{2} P^{\prime}-A \frac{\partial^{3} P^{\prime}}{\partial \tau^{3}}=\frac{B}{2} \frac{\partial^{2} P^{\prime 2}}{\partial \tau^{2}}, \\
& \sigma=\frac{z}{F}, \\
& A=\alpha F, \\
& B=\frac{\beta \omega^{*} P_{0} F}{\rho_{0} c_{0}^{3}}, \\
& \tau=\omega^{*}\left(t-\frac{z}{c_{0}}\right), \\
& P^{\prime}=\frac{P}{P_{0}},
\end{aligned}
$$

where $P_{0}$ is the acoustic pressure field amplitude on the surface of the transducer and $c_{0}$ is the speed of sound in the medium. The $z$ axis is taken along the propagation direction of the acoustic beam. The parameter $\alpha$ accounts for thermal and viscous losses, $\beta$ is the coefficient of nonlinearity, and $\rho_{0}$ is the density of the liquid. The transverse Laplacian operator $\nabla_{\perp}^{2}$ accounts for wave-front curvature associated with diffraction. The solution to Eqs. (18) along with a measurement of $P_{0}$ provides the counterpart to the results obtained from light-diffraction theory. One can determine the pressure at the focus as a function of input power by measuring the diffracted light and compare the results with those determined independently by either the theory of Debye or solutions to the KZK equation to test the modified Raman-Nath model.

\section{RESULTS AND DISCUSSIONS}

A PZT-4 (Staveley Sensors, East Hartford, CT) spherical concave piezoelectric transducer was suspended in a rectangular container with glass windows on the sides. Both the convex and concave sidcs of the transducer were silver coated. A three-point support on the bottom served as one electrical contact, and three springy metal wires provided contact on the top or concave side of the transducer. A Hewlett-Packard 651B oscillator is used to drive the transducer. The electrical signal from the oscillator is amplified by a homemade of amplifier before being applied to the transducer. There is no impedance matching layer on either concave or convex side of the transducer. The liquid used is distilled water with specific resistance over 10 $\mathrm{M} \Omega / \mathrm{cm}$; the value of $(\partial \mu / \partial P)_{s}$ is equal to $14.66 \times 10^{-11}$ $\mathrm{Pa}^{-1} . \alpha$ is equal to 1.50 , and $\beta$ is equal to 6.0 . The transducer with the series resonance frequency at $2.15 \mathrm{MHz}$ has a radius of curvature of $2.54 \mathrm{~cm}$, a thickness of $0.028 \mathrm{~cm}$, and an aperture angle of $26.66^{\circ}$.

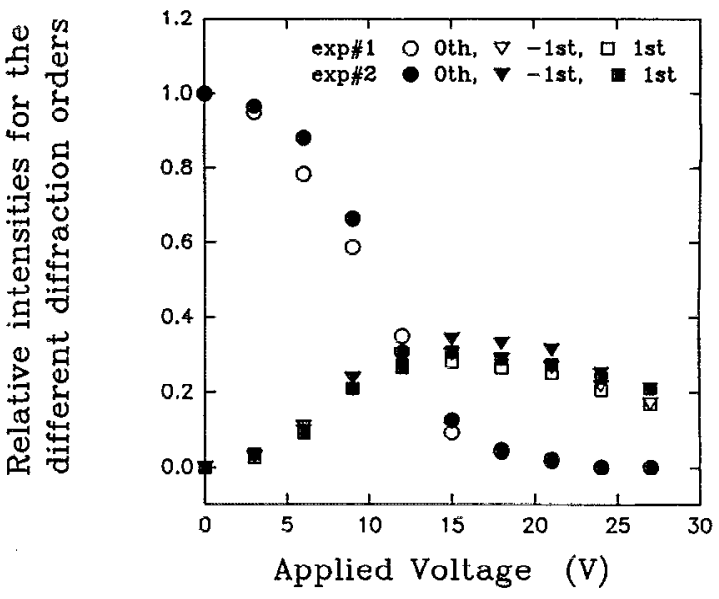

FIG. 1. Measured light intensities relative to the intensity of the undiffracted beam for the zeroth, positive, and negative first diffraction orders vs the applied voltage.

Using the method described in the Appendix, we are able to determine the pressure on the surface of the transducer. Then either Debye's formula or the KZK equation was used to calculate the pressure in the focal plane.

The light from a He-Ne laser with output power of 0.8 $\mathrm{mW}$ and beam size of $0.8 \mathrm{~mm}$ passes through the windows on the container and through the focal plane of the transducer. The beam is perpendicular to the propagation direction of the sound beam. The vertical diffraction pattern, parallel to the propagation direction of the acoustic wave, is measured using a photodiode (UDT3DP1). The output of the photodiode was amplified so that the electrical signal corresponding to the undiffracted laser beam was about 8 V. The photodiode was scanned through the beam spot to collect the optical signal, i.e., the diffraction intensities for the first three orders, and the signal was recorded with a $X-Y$ recorder. Figure 1 shows the measured light-diffraction intensities for the zeroth, negative, and positive first diffraction orders versus the applied voltage. Note that the higher orders of diffraction are observed but not used in our analysis because no additional information is provided by these higher orders.

By the method described in the Appendix, we obtained the relationship between the surface pressure amplitude and the applied voltage to the transducer. Thus we have a relation between the surface pressure and the various light intensities. From the light intensities, we calculated [using Eq. (8)] the Raman-Nath parameter and $a_{2}$. We found that an $a_{2}$ of approximately 0.04 best represents our data. Via Eq. (17), the Raman-Nath parameter gave the peak pressure in the focal plane.

Figures 2(a), 2(b), and 2(c) show the pressure amplitudes obtained from our modified model for the zeroth, negative, and positive first order of the diffracted light, respectively. The following can be observed: The agreement between the modified Raman-Nath model [Eqs. (8) and (17)] and Debye's theory [Eq. (18)] and the calculation using the KZK equation is good and results obtained 

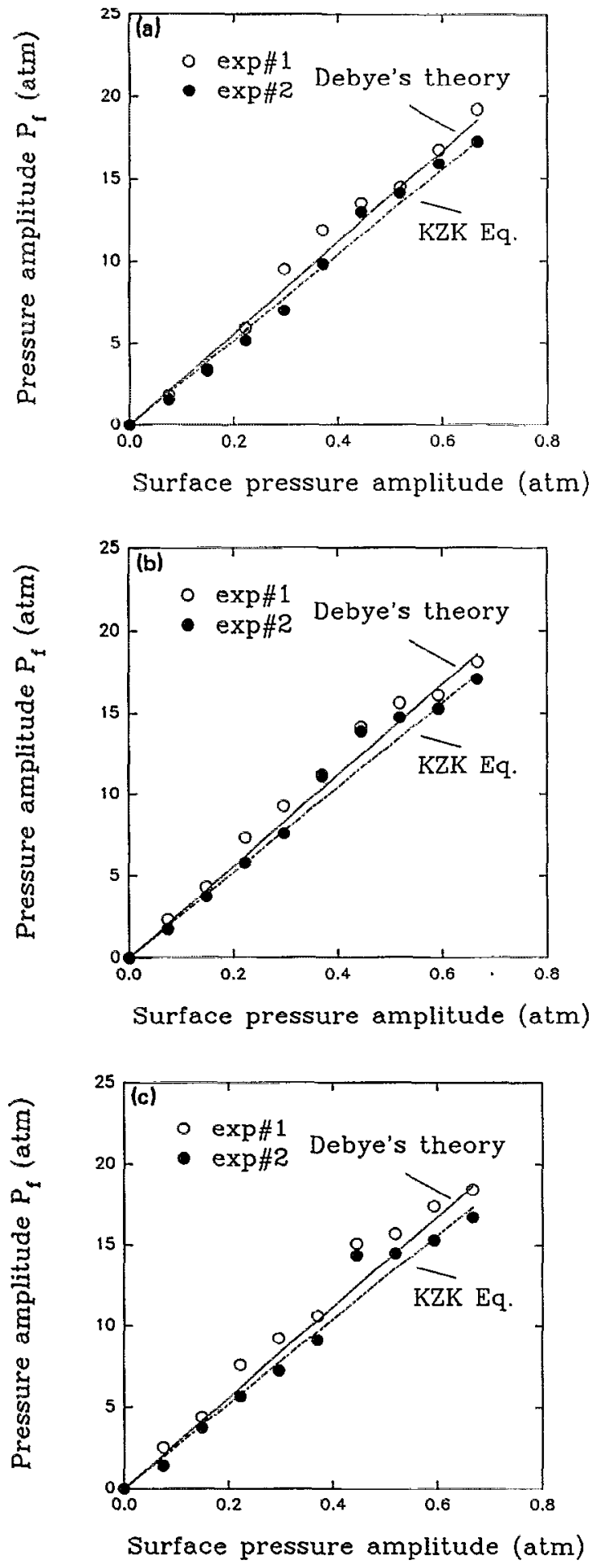

FIG. 2. Pressure at the focal point vs the surface pressure on the transducer calculated from (a) the zeroth-order diffraction, (b) the positive first, (c) the negative first, and comparison with the results from Debye's theory and the KZK equation assuming an amplitude of the second harmonic equal to $4 \%$ of the fundamental. The dots are the measured intensities of the diffracted light. The solid line is calculated from Debye's theory. The dashed line is the result from the KZK equation. In the KZK case, only the fundamental pressure is plotted.

from all the three different diffraction orders are consistent. The peak pressure according to the KZK equation is less than what is obtained from Debye's theory because absorption and nonlinearity are being considered. Because the

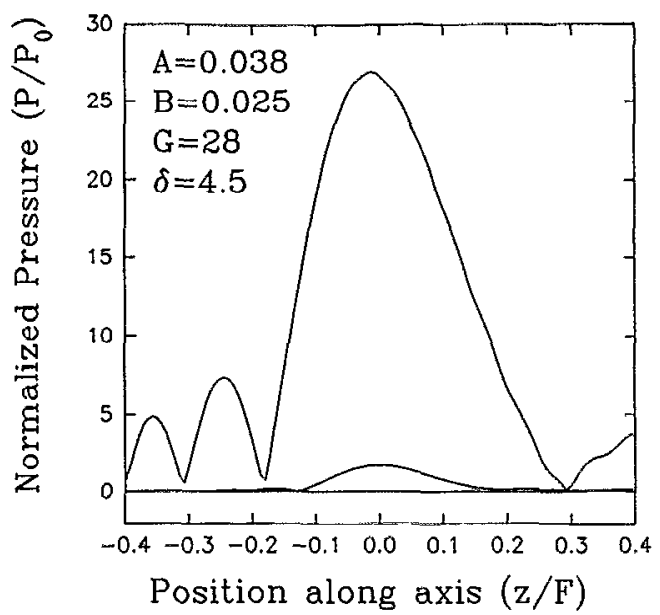

FIG. 3. The axial pressure distribution (for the first two harmonics) calculated from the KZK equation with a value of 0.025 for the nonlinearity parameter $B$.

changes in intensity of the diffracted light are smaller for larger amplitudes, the measured pressure amplitudes from the different diffraction orders are more uncertain than those obtained for smaller amplitudes. When the RamanNath parameter is around 2 (a pressure of about $12 \mathrm{~atm}$ ), the diffraction intensity of the zeroth order is very close to zero, but intensities of both the negative and positive first orders are close to maximum. Therefore, the pressure amplitude determined by the diffracted light of the zeroth order is not as accurate as that determined by the positive and negative first orders. Note that in Figs. 2(a)-2(c), we used our effective interaction length to replace the "standard" light-sound interaction length.

Following the method developed by Hart, ${ }^{23}$ we solved the $\mathrm{KZK}$ equation numerically with all of the parameters determined by our system. The acoustic gain coefficient $G$ is 28 , the loss coefficient $A$ is 0.0038 , and the nonlinearity constant $B$ is in the range from 0 to 0.025 , corresponding to a pressure at the source of up to about $1 \mathrm{~atm}$. In order to determine the proper value of the parameter $\delta$, which controls the convergence of the program, we calculated the normalized pressure distribution along the propagation direction and found that $\delta$ should be equal to 4.5 . With this value for $\delta$, we performed calculations of the axial and radial pressure distributions by including the first four harmonics. For $B=0.025$, the calculated axial pressure distribution (along the propagation direction) is shown in Fig. 3, and the transverse distribution (in the focal plane) is shown in Fig. 4. For low pressures, only the fundamental harmonic is important. In this case, Debye theory obtains [Eq. (13)]. For the higher pressures, higher harmonics show up as shown in Figs. 3 and 4.

The solutions to the KZK equation with the material constants of water and the pressure amplitude at the surface of the transducer as the only input data give us an approximate way to calculate $a_{2}$, the amount of second harmonic. From Fig. 4, the ratio of the pressure amplitude of the second harmonic so that of the fundamental is found 


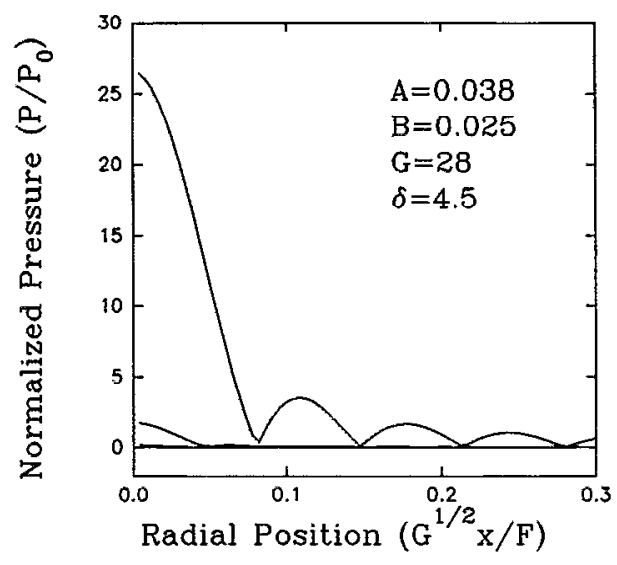

FIG. 4. The transverse pressure distribution (for the first three harmonics) in the focal plane calculated from the KZK equation with a value of 0.025 for the nonlinearity parameter $B$.

to be 0.065 . This is not equal to $a_{2}$, however, since the region where the second harmonic is important is smaller than the region in which the fundamental contributes to the light scattering; the second harmonic is focused to a smaller spot. In order to calculate the effective $a_{2}$, one has to perform the integration of Eq. (9) with $P(x)$ being the pressure distribution of the second harmonic. An approximate value can be obtained by comparing the width at half maximum of the central peak for the second-harmonic pressure distribution with the corresponding width of the fundamental. Doing this gives an effective $a_{2}$ of 0.04 . This is in good agreement with our observation of the asymmetry in the light-diffraction patterns. ${ }^{15}$

By solving the KZK equation, we also obtain the phase factors $\delta_{j}(r)$. To assess the approximate influence of these phase factors, one can compare $P_{j}(r) \cos \left[\delta_{j}(r)\right]$ with $P$. When the integration in Eq. (14) is performed, one can argue that only the real part plays a rolc. Calculations show that the difference between $P_{j}(r)$ and $P_{j}(r) \cos \delta_{j}(r)$ is negligible, and thus we can ignore the phase factors. Therefore, the effective interaction length calculated with the numerical results from the KZK equation is not very different from that obtained from the theory of Debye in the pressure range that we are concerned with.

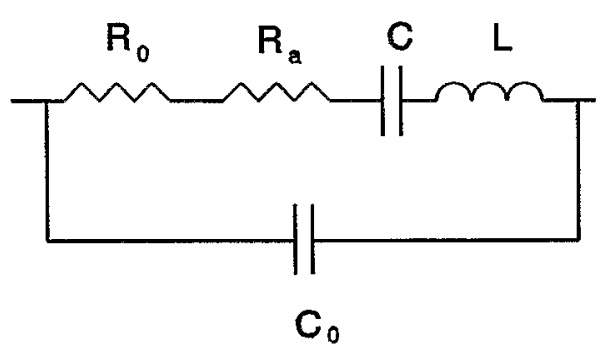

FIG. 5. The equivalent circuit of a focusing transducer. $R_{T}=R_{0}$ $+R_{A}$, where $R_{0}$ represents the load caused by the supports and the piezoelectric ceramic and $R_{A}$ represents load of the surrounding medium.

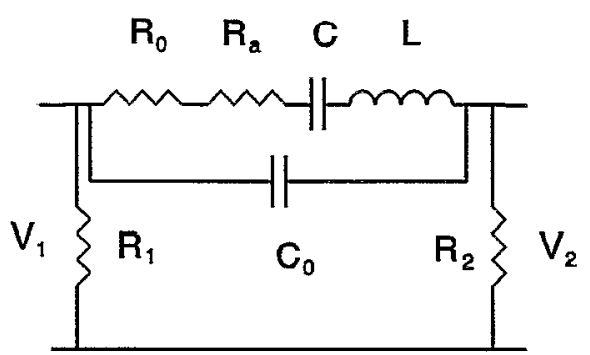

FIG. 6. The gate circuit measuring the impedance of the transducer.

\section{CONCLUSIONS}

We have shown that Raman-Nath theory, as modified by the introduction of an effective interaction length, can be used to determine the pressure amplitude in the focal plane of a focusing transducer with aperture angles of about $30^{\circ}$, and the results obtained from different diffraction orders are consistent. This is a quite unexpected result since it was shown by Nagai $^{13}$ that Raman-Nath theory fails for nonplanar wave fronts. However, in the focal plane the wave fronts can be approximated by plane waves, and
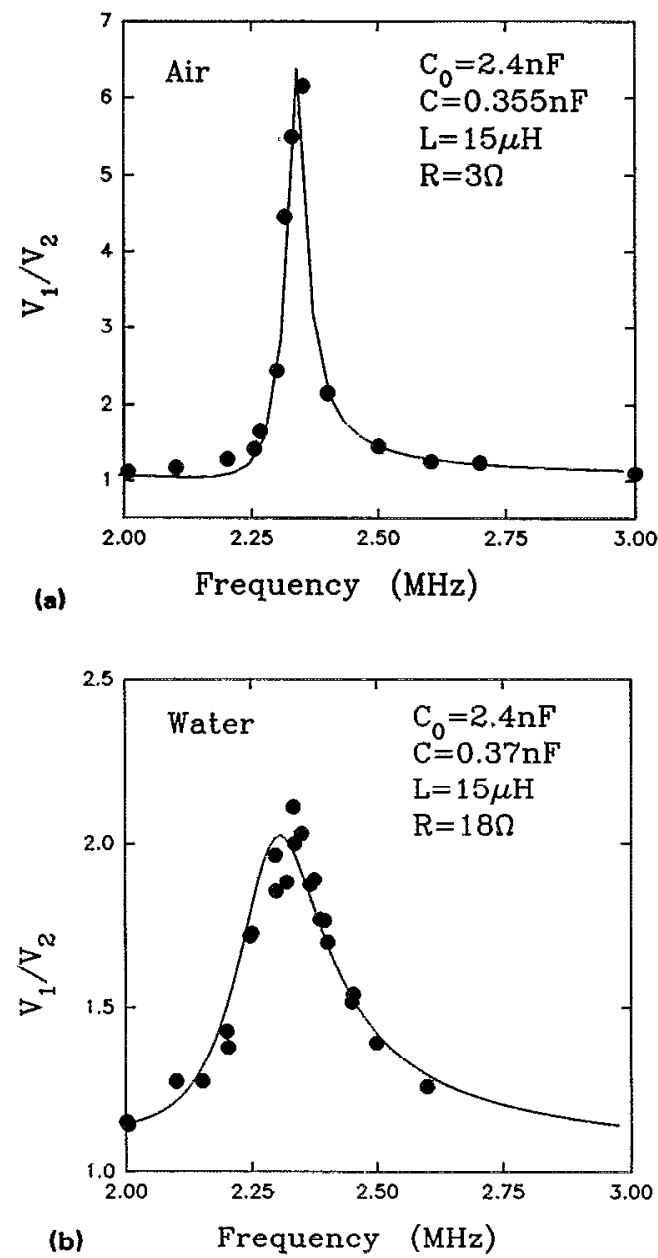

FIG. 7. Measured ratio of $V_{\text {out }} / V_{\text {in }}$ vs frequency (a) in air and (b) in water and the fitted curves. 
consequently the usual analysis can be made. We have shown that as long as the nonlinearity is not too large, the experimental results follow closely the calculated results and that the light diffraction contains enough information to calculate the second-harmonic content. In principle, it is possible that the contribution to the acoustic wave of the higher than the second-order harmonic can be obtained. The importance of this light-diffraction technique seems to be twofold. It can be used as a probe to measure the pressure field of a focusing transducer without disturbing the acoustic field in contrast to a physical sensor. In addition, this technique might make an experimental analysis of large-aperture-angle transducers possible where so far theories are not well developed.

\section{APPENDIX: CALCULATION OF THE SURFACE PRESSURE OF A TRANSDUCER}

To obtain the pressure at the focus, one needs to know the pressure on the surface of the acoustic transducer. This pressure can be calculated from the electrical properties of the transducer and the power supplied to it. As shown by Zelenka, ${ }^{24}$ the electric properties of a piezoelectric transducer can be modeled by an equivalent electric circuit consisting of resistors, capacitors, and an inductor. Usually, this model deals with a flat-plate transducer. We apply it to the focusing transducer based on the argument that the diameter of the transducer is much larger than the thickness. The circuit diagram is shown in Fig. 5. The total electrical impedance of the transducer at a particular frequency is found to be

$$
Z_{T}(\omega)=\frac{\omega L-1 / \omega C-j R_{T}}{\omega C_{0} R_{T}+j\left[\omega C_{0}(\omega L-1 / \omega C)-1\right]} .
$$

After measuring the impedance at different frequencies, the parameters of the model can be obtained by a least-squares fit. When the transducer is submerged in the liquid, $R_{T}$ equals $R_{A}$, the equivalent electrical resistance due to the acoustic load presented by the liquid to the transducer plus $R_{0}$, the resistance due to the supports. With the transducer suspended in air, $R_{T}$ is cqual to the same support resistance plus the radiation resistance of the air. But since this resistance is proportional to $\rho c$, with $\rho$ the density and $c$ the speed of sound of the medium, it is negligible in air. Thus we essentially measure $R_{0}$ in the latter case. Since the acoustic energy radiated into the liquid is equal to the electrical energy $E$, dissipated in $R_{A}$, one obtains

$$
E=\frac{V^{2}}{R_{a}}=\frac{P^{2} A}{2 \rho_{0} c},
$$

where $P$ is the pressure amplitude at the surface of the transducer, $\rho_{0}$ is the density of the liquid, $V$ is the calculated voltage driving $R_{A}$, and $A$ is the surface arca of the transducer.

We set up a gate circuit as shown in Fig. 6. The ratio of output and input voltages can be expressed

$$
\frac{V_{1}}{V_{2}}=\frac{Z_{\|}+R_{2}}{R_{2}}
$$

where

$$
\frac{1}{Z_{\|}}=\frac{1-\omega C_{0}(\omega L-1 / \omega C)+j \omega C_{0} R_{0}}{R_{0}+j(\omega L-1 / \omega C)}
$$

By measuring the input and output voltages at the different frequencies and using the least-squares fitting method, we obtain the values for the resistance, the capacitances, and the inductance. Therefore, the surface pressure can be determined. The resultant fitted curves are shown in Figs. 7 (a) and $7(\mathrm{~b})$, which show the data in air and water, respectively.

${ }^{1}$ A. Korpel, Proc. IEEE 69, 48 (1981).

${ }^{2}$ K. Van Den Abeele and O. Leroy, J. Acoust. Soc. Am. 88, 2298 (1990).

${ }^{3}$ P. Kwiek, J. Acoust. Soc. Am. 86, 2261 (1989).

${ }^{4}$ J. A. Nissen, E. Bodegom, L. C. Brodie, and J. S. Semura, Phys. Rev. B 40, 6617 (1989).

${ }^{5}$ C. V. Raman and N. S. Nath, Proc. Indian Acad. Sci. A 2, 406 (1935).

${ }^{6}$ R. Mertens, Meded. K. Vlaam. Acad. Wet. Lett. Schone Kunsten Belg. Kl. Wet. 12, 1 (1950).

${ }^{7}$ J. C. Berlinghieri and B. D. Cook, J. Acoust. Am. 58, 823 (1975).

${ }^{8}$ A. Korpel, J. Opt. Soc. Am. 69, 295 (1979).

${ }^{9}$ W. G. Mayer and T. D. K. Ngoc, Acoust. Lett. 9, 171 (1980).

${ }^{10}$ O. Leroy and J. M. Claeys, Acustica 55, 21 (1984).

${ }^{11}$ W. R. Klein and B. D. Cook, IEEE Trans. Sonics Ultrason. 14, 123 (1976).

${ }^{12}$ R. Reibold, Acustica 36, 214 (1976).

${ }^{13}$ S. Nagai, J. Acoust. Soc. Jpn. 7, 229 (E) (1986).

${ }^{14}$ B. D. Cook, J. Acoust. Soc. Am. 37, 172 (1965).

${ }^{15}$ K. L. Zankel and E. A. Hiedemann, J. Acoust. Soc. Am. 31, 44 (1959).

${ }^{16}$ L. R. Hargrove, J. Acoust. Soc. Am. 43, 847 (1968).

${ }^{17}$ R. Reibold and P. Kwiek, Acustica 70, 223 (1990).

${ }^{18}$ H. A. Kashkooli, P. J. Dolan, and C. W. Smith, J. Acoust. Soc. Am. 82, 2086 (1987).

${ }^{19}$ P. Debye, Ann. Phys. (N.Y.) 30, 755 (1909).

${ }^{20}$ T. O'Neil, J. Acoust. Soc. Am. 21, 516 (1949).

${ }^{21}$ V. M. Levin, O. T. Lobkins, and R. G. Maev, Sov. Phys. Acoust. 33, 87 (1987).

${ }^{22}$ T. Hart and M. F. Hamilton, J. Acoust. Soc. Am. 84, 1488 (1988).

${ }^{23}$ T. Hart, M. S. thesis, The University of Texas at Austin, 1987.

${ }^{24} \mathrm{~J}$. Zelenka, Piezoelectric Resonators and their Applications (Elsevier, New York, 1986). 